\title{
Association of strength, muscle balance, and atrophy with pain and function in patients with degenerative spondylolisthesis
}

\author{
Tania Inés Nava-Bringas ${ }^{\mathrm{a}, *}$, Isabel Ramírez-Mora ${ }^{\mathrm{b}}$, Roberto Coronado-Zarco ${ }^{\mathrm{a}}$, \\ Salvador Israel Macías-Hernández ${ }^{\mathrm{a}}$, Eva Cruz-Medina ${ }^{\mathrm{a}}$, Aurelia Arellano-Hernández ${ }^{\mathrm{a}}$, \\ Mario Hernández- López ${ }^{\mathrm{a}}$ and S.R. León-Hernández ${ }^{\mathrm{c}}$ \\ ${ }^{a}$ Department of Spine Rehabilitation, National Institute for Rehabilitation, México City, Mexico \\ ${ }^{\mathrm{b}}$ Department of Imaging, National Institute for Rehabilitation, México City, Mexico \\ ${ }^{\mathrm{c}}$ Department of Research, National Institute for Rehabilitation, México City, Mexico
}

\begin{abstract}
.
PURPOSE: To analyze the relationship of strength, muscular balance, and atrophy with pain and function in patients with degenerative spondylolisthesis.

STUDY DESIGN: Transversal, descriptive, and observational.

PATIENTS AND METHODS: Institutional review board approval was obtained for this study. Twenty six patients ages 50 years and older, with degenerative spondylolisthesis at L4-L5. Measurements included Pain Visual Analogue Scale scores (VAS), Oswestry Disability Index scores (ODI), and isokinetic trunk testing; assessment of multifidus atrophy and spinal stenosis was performed by Magnetic Resonance Imaging (MRI).

STATISTICS: Statistical analysis was performed using SPSS version 17.0 software for Windows. Pearson's correlation was used to ascertain the correlation between variables. ANOVA with analysis of covariance was used to determine the correlation between the remainder variables. Significance was set at $p<0.05$.

RESULTS: Of the 26 patients studied, with an average age of $60.23 \pm 7.6$ years, 20 had grade I spondylolisthesis and 6 were grade II. Correlation between the ODI scores and spondylolisthesis grading was significant $(r=0.576, p=0.005)$; correlation between agonist/antagonist ratio in the isokinetic test (predominant extensor muscles over flexors) with the ODI scores was also significant $(r=0.446, p=0.02$ ), regardless of spinal stenosis. No correlation was found between functionality and pain with strength or multifidus atrophy.
\end{abstract}

CONCLUSION: Muscle trunk imbalance with predominance of extensor over flexor muscles is associated with functional disability. Rehabilitation programs should be designed to improve muscle balance rather than muscle strength alone.

Keywords: Multifidus atrophy, degenerative spondylolisthesis, pain, functionality, strength

\section{Introduction}

Degenerative spondylolisthesis (DS) is common in

*Corresponding author: Tania Inés Nava-Bringas, Instituto Nacional de Rehabilitación, Rehabilitación de Columna, Av. MéxicoXochimilco 289, Col. Arenal de Guadalupe, Tlalpan 14389, México. Tel.: +52 555999 1000; E-mail: tanianava@gmail.com. patients over 50 years of age and is four fold more frequent in women with a multifactorial etiology [1,2].

The initial event comprises intervertebral disc degeneration with narrowing of the disc space and arthritis of the facet joints. Degenerative changes that occur later with subchondral sclerosis, ossification of ligaments, and hypertrophic facet arthrosis provide a natural tendency to re-stabilize the segment $[3,4]$.

Symptoms of mechanical low back pain (LBP) asso- 
Table 1

Mean descriptive data for height, weight, and body mass index (BMI)*

\begin{tabular}{lccrr}
\hline Parameter & Min & Max & Mean & \multicolumn{1}{c}{ SD } \\
\hline Age (years) & 50 & 81 & 60.2 & 7.64 \\
Weight (kg) & 49.8 & 95.4 & 71.3 & 12.29 \\
Height (cm) & 140.00 & 177.00 & 155.30 & 9.24 \\
BMI & 20.94 & 39.67 & 29.63 & 4.70 \\
Disease evolution time (months) & 1.00 & 24.00 & 12.26 & 7.23 \\
\hline
\end{tabular}

$* n=26$. Min $=$ Minimun value; Max = Maximum values; $\mathrm{SD}=$ Standard deviation; BMI $=$ Body mass index.

ciated to degenerative changes may develop, as well as radicular pain from nerve root compression and neurogenic claudication produced by concomitant spinal stenosis [5-10].

Panjabi defined clinical instability associated with degenerative disease as an abnormal movement that occurs in the "neutral zone" (NZ) within the total range of motion $[11,12]$. In DS, the initial phase of segmental instability tends to increase the NZ, but over time degenerative changes cause a reduction in the NZ. Both instances are undesirable and are associated with pain and dysfunction $[11,12]$.

The role of trunk muscles has been evaluated in chronic LBP wheremultifidus atrophy and delayed activation have been observed, with pain reduction after core stability training. These muscles changes have not been studied in DS [13-15].

Therefore the aim of this study was to analyze the relationship of trunk strength and muscular balance, as well as multifidus atrophy with functionality and pain in patients with DS.

\section{Materials and methods}

\subsection{Study design}

An observational, cross-sectional study was performed.

\subsection{Patients}

Sample size measurement showed a requirement for 23 patients to ensure a correlation coefficient of at least 0.50 among the main variables, with an error rate $<$ 0.05 and a statistical power of $80 \%$. Patients accepted in the study were 50 years of age and older, with degenerative spondylolisthesis at L4-L5, attending the Spinal Rehabilitation Service of the National Institute for Rehabilitation (Mexico City). They were without treatment, and agreed to participate through informed consent. Patients with a history of lumbar surgery, rheumatic diseases, cauda equine symptoms, ischemic heart disease, or diabetic polyneuropathy were excluded.

\subsection{Evaluations}

Data on evolution time, clinical presentation (mechanical back pain, radiculopathy or neurogenic claudication), use of analgesics, hypertension, diabetes, history of smoking, Pain Visual Analogue Scale (VAS) scores for pain and Oswestry Disability Index (ODI) [16] scores for disability were collected.

Isokinetic trunk test: Average strength, total work and the agonist-antagonist relationship (ratio) were chosen as measurements of muscle strength and balance.

Lumbar spine radiography: Radiographic studies were used to determine spondylolisthesis grades according to Meyerding's Classification and sagittal displacement (in millimeters [mm]) in flexion-extension radiographs to analyze intersegmental instability.

Lumbar Magnetic Resonance Imaging (MRI): Spinal stenosis, multifidus atrophy, fatty infiltration, and degenerative changes at the intervertebral disc were determined by MRI.

\subsection{Procedures}

Clinical evaluation, VAS and ODI scores, isokinetic tests, and radiographic measurements were performed by a single physician with extensive clinical experience and with training for the use of isokinetic testing devices.

Isokinetic evaluation: A 5-min warm-up was performed on a lower body ergometer at a resistance level of $60 \mathrm{rpm}$. Following warm-up, subjects were placed in the Cybex Torso Flexion-Extension isokinetic dynamometer (Cybex Back Systems). Vertical, horizontal axis and upper and lower body positions were stabilized for safety and data accuracy by locking pads and belts. A $30^{\circ}$ arc of total movement was set using range of motion stops $\left(10^{\circ}\right.$ of extension and $20^{\circ}$ for flexion). Five maximal repetitions at $60^{\circ}$ Section 15 maximal repetitions at $90^{\circ} \cdot \mathrm{sec}$ were employed for data collection. Consistent verbal commands were given, with no visual feedback allowed during the testing protocol. The reliability of the Cybex isokinetic trunk system has been previously established and published [17].

MRI measurements: These measurements were performed by a single highly trained radiologist, who did 
Table 2

Comparative mean values between the 3 clinical presentation in patients with DS (LBP, radiculopathy or neurogenic claudication)

\begin{tabular}{lcccc}
\hline Parameter & LBP $n=20$ & Radiculopathy $n=4$ & Neurogenic claudication $n=2$ & $F$ \\
\hline Age (years) & $61.3 \pm 7.8$ & $54.7 \pm 7.0$ & $60.5 \pm 3.5$ & 1.248 \\
Evolution time, (months) & $11.4 \pm 7.1$ & $16.5 \pm 9.0$ & 0.306 \\
VAS “back" (mm) & $63.2 \pm 17.99$ & $57 \pm 15.53$ & $83.00 \pm 0.00$ & 0.8 \\
Oswestry (\%) & $31.85 \pm 16.26$ & $31.00 \pm 13.31$ & $37.00 \pm 15.55$ & 0.461 \\
\hline
\end{tabular}

LBP $=$ Low back pain. *ANOVA test.

Table 3

Function and pain assessments*

\begin{tabular}{lcccc}
\hline & Min & Max & Mean & SD \\
\hline VAS "back pain" score $(\mathrm{mm})$ & 24 & 96 & 63.8 & 18.06 \\
VAS “sciatic pain" score $(\mathrm{mm})$ & 0 & 97 & 53.9 & 27.24 \\
Oswestry index $(\%)$ & 2 & 66 & 32 & 15.30 \\
\hline
\end{tabular}

$* n=26$. Min = Minimun value; Max = Maximum values; $\mathrm{SD}=$ Standard deviation; VAS $=$ Visual analogue scale.

Table 4

Intergender differences between mean values for isokinetic trunk test evaluations

\begin{tabular}{lrrc}
\hline Parameter & Females & Males & $p$ (2-tailed) \\
\hline Flexor strength $(\mathrm{Nm})$ & 71.95 & 152.83 & 0.001 \\
Extensor strength $(\mathrm{Nm})$ & 59.5 & 127.0 & 0.001 \\
Strength ratio F/E & 0.83 & 0.82 & 0.925 \\
Flexor total work $(\mathrm{J})$ & 286.60 & 752.66 & 0.001 \\
Extensors total work $(\mathrm{J})$ & 220.60 & 540.66 & 0.001 \\
Total work ratio F/E & 1.30 & 0.74 & 0.589 \\
\hline
\end{tabular}

$* \mathrm{~F} / \mathrm{E}=$ Flexor/extensor; $\mathrm{Nm}=$ Newtons/meters; $\mathrm{J}=$ Joules.

not know the clinical status of the patients. MRI was performed on a $\mathrm{GE}^{\mathrm{R}} 1.5 \mathrm{~T} \mathrm{Hdx}$, with conventional T1 and $\mathrm{T} 2$ sequences and axial scans. AP sagittal diameter was measured for stenosis. Cross-sectional area in $\mathrm{mm}^{2}$ at L3-L4, L4-L5, and L5-S1 were evaluated for multifidus atrophy (Fig. 1). Degree of fat infiltration was established by the same classification used by Kjaer [18] in 2007: "normal" for estimates of 0-10\% fat within the muscle; "slight" for $10-50 \%$ fat, and "severe" for $>50 \%$ fat infiltration. Degenerative changes at the intervertebral disc were described according to the Pfirrmann'sclassification [19].

\subsection{Data analysis}

Statistical analysis was performed using SPSS version 17.0 software for Windows. Based on the data obtained, a descriptive analysis of variables of interest was performed using centralization and dispersion measures. The normality of the quantitative variables was verified using the Kolmogorov-Smirnov test; for variables following a normal distribution, further analysis was performed using parametric tests. Pearson's correlation test was performed to ascertain the degree of the relation between VAS, ODI, multifidus atrophy
Table 5

Intergender differences between mean values of multifidus crosssectional areas*

\begin{tabular}{llcc}
\hline Intervertebral level & Males & Females & $p$ \\
\hline L3-L4 total $\left(\mathrm{mm}^{2}\right)$ & 806.1 & 691.2 & 0.14 \\
L3-L4 right $\left(\mathrm{mm}^{2}\right)$ & 411 & 362.9 & 0.26 \\
L3-L4 left $\left(\mathrm{mm}^{2}\right)$ & 395.1 & 328.2 & 0.10 \\
L4-L5 total $\left(\mathrm{mm}^{2}\right)$ & 1.133 & 873.6 & 0.03 \\
L4-L5 right $\left(\mathrm{mm}^{2}\right)$ & 571.8 & 445.8 & 0.05 \\
L4-L5 left $\left(\mathrm{mm}^{2}\right)$ & 561.3 & 427.8 & 0.02 \\
L5-S1 total $\left(\mathrm{mm}^{2}\right)$ & $1,246.8$ & $1,039.1$ & 0.17 \\
L5-S1 right $\left(\mathrm{mm}^{2}\right)$ & 618.6 & 528.9 & 0.26 \\
L5-S1 left $\left(\mathrm{mm}^{2}\right)$ & $628.1 \mathrm{~mm}^{2}$ & $510.2 \mathrm{~mm}^{2}$ & 0.11 \\
\hline
\end{tabular}

$\mathrm{mm}^{2}=$ square milimeters. *Multifidus cross sectional areas were evaluated at three levels for both sides of spinal segments, and a single total area was determined with the sum of both values.

and strength, and subsequently ANOVA with analysis of covariance was applied to determine the relationship between main variables with control of the remainder of the variables studied. Due to intergender differences of sample size, statistical analysis was first performed for men and women together, as a single group, and then for women only; due to low statistical power this was not possible for men. For this study, significance was set at 0.05 .

\section{Results}

Twenty six patients were included (20 females and 6 males). Main descriptive data are reported in Table 1. Clinical presentation was LBP in $77 \%$ (15 females 5 males), radiculopathy in $15 \%$ (4 females), and $8 \%$ with neurogenic claudication (1 male and 1 female), without differences among groups (see Table 2). Functional assessments are shown in Table 3. Intergender differences were found only for back pain. VAS scores for females were $59.2 \pm 17.4 \mathrm{~mm}$, and $79.1 \pm 10 \mathrm{~mm}$ for males $(p=0.015)$.

Of the 26 patients, $23 \%$ had a history of hypertension and $7.7 \%$ had diabetes mellitus; $42.3 \%$ had a history of smoking and $50 \%$ of patients said to have taken analgesics within the previous month.

According to Meyerding's classification, 20 cases $(77 \%)$ were grade I and six $(23 \%)$ were grade II. 


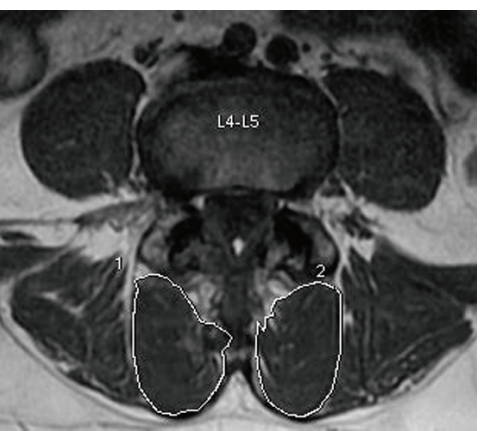

(a)

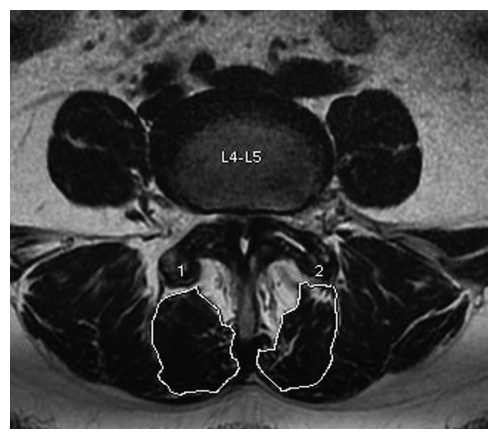

(b)

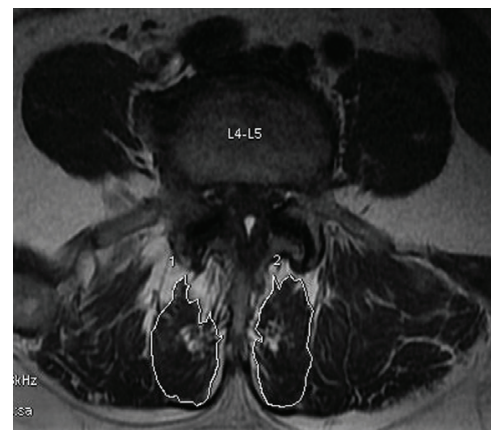

(c)

Fig. 1. MRI images of outlined multifidus cross sectional areas (in $\mathrm{mm}^{2}$ ) at L4-L5, showing patterns of fat infiltration. 1a. Normal tissue, without fat infiltration. 1b. Slight fat infiltration (less than 50\%). 1c. Severe fat infiltration (over 50\%).

Table 6

Differences in strength and multifidus cross-sectional areas between women with clinical manifestations of LBP or radiculopathy

\begin{tabular}{lccl}
\hline Parameter & Low back pain & Radiculopathy & \multicolumn{1}{c}{$p$} \\
\hline Flexor strength $(\mathrm{Nm})$ & $65.2 \pm 17.2$ & $82.5 \pm 15.0$ & 0.08 \\
Extensor strength $(\mathrm{Nm})$ & $51.2 \pm 17.2$ & $82.7 \pm 18.0$ & 0.005 \\
Extensor total work $(\mathrm{J})$ & $162.5 \pm 112.3$ & $395.7 \pm 208.3$ & 0.007 \\
MCSA at L4-L5 $\left(\mathrm{mm}^{2}\right)$ & $801.1 \pm 243.7$ & $1001.2 \pm 214.2$ & 0.07 \\
MCSA at L5-S1 $\left(\mathrm{mm}^{2}\right)$ & $932.3 \pm 234.7$ & $1289.2 \pm 390.2$ & 0.03 \\
\hline
\end{tabular}

MCSA: multifidus cross-sectional areas, $\mathrm{Nm}=$ Newtons/meter; $\mathrm{J}=$ Joules. $\mathrm{mm}^{2}=$ square millimeters.

Fromflexion-extension radiographs, sagittal displacements were measured and found to be: $2 \mathrm{~mm}$ for $46.2 \%$ of the patients, $3 \mathrm{~mm}$ for $34 \%$, $4 \mathrm{~mm}$ for $11.5 \%$ and $1 \mathrm{~mm}$ in only $7.7 \%$ of the cases.

Isokinetic test results are depicted in Table 4. Intergender differences in strength and total work were clearly significant.

Multifidus atrophy measurements are shown in Table 5; they were not influenced by weight, BMI, history of smoking, or DM, nor with degree of spondylolisthesis, or disc degeneration.

According to Pfirrmann's classification, one half $(53.8 \%)$ of patients had degenerative disc changes corresponding to grade IV, $23.9 \%$ were classified as grade III, $11.5 \%$ asgrade V, and $10.8 \%$ as grade II.

A total of $73.1 \%$ of the patients had severe fatty infiltration (grade III), followed by $15.4 \%$ with mild infiltration (grade II), and $11.5 \%$ were considered normal (grade I). Female participants had markedly higher prevalence rates of fat; intergender differences were significant ( $p=0.002$, OR. 45.0 IC 3.3-6.3); these observations were not influenced either by weight ( $p=$ $0.47)$ or by BMI ( $p=0.55)$. See Figs $1(\mathrm{a})-(\mathrm{c})$.

A positive relationship was found between degree of spondylolisthesis and ODI scores: analysis for men and women together, as a single group, was $r=0.576$, $p=0.005$ and, $r=0.368, p=0.015)$ for women only, with a higher average of disability in patients with spondylolisthesis grade II compared with those with grade I; significance was reaffirmed in the covariance analysis performed to control for spinal stenosis $(r=$ $0.524, p=0.002$ ).

A positive correlation was also found for muscle imbalance (flexor/extensor ratio); thus, the prevalence of trunk extensors was related to a higher rate of disability (men and women $r=0.446, p=0.02 ; r=0.422$, $p=0.04$, for women only).

An interesting finding was that average strength, total work force, and multifidus atrophy at the L4-L5 and L5-S1 segments were significantly lower in women with LBP compared to women with radiculopathy (see Table 6).

No relationship was found between pain scores (VAS) or functionality scores (ODI) with weight, BMI, degree of disc degeneration, intersegment displacement observed in the flexion-extension radiographs, strength or total work force, and multifidus atrophy.

\section{Discussion}

Degenerative spondylolisthesis is associated with a wide range of symptoms, from LBP to neurogenic claudication. L4-L5 vertebral space is affected 6-9 times more frequently thanany other spinal level [20], thus the focus of this research.

One of the aims of the present study was to determine if differences in pain and dysfunction might be related with multifidus atrophy, as has been reported for chronic LBP [20-22]. In these cases, LBP was unspecific and patient population was diversified.

Fat infiltration results were found to be consistent with those reported by Kjaer [20] in which women 
showed greater fat infiltration, regardless of weight or BMI and are more likely related to intergender differences in body composition.

A particularly interesting finding was the association between multifidus trophism and clinical presentation that allowed us to differentiate between patients with LBP and radiculopathy. We do however caution that it is necessary to have a larger patient population (including a larger number of males) to confirm these differences and determine the extent of their clinical implication, if any.

Spondylolisthesis symptoms are associated with degenerated and subluxated facet joints, segmental instability that causes tension of facet joint capsule and ligaments, as well as overuse of stabilization muscles [20], which could explain why these structures differ in trophism when there is greater potential for damage to neural structures as in radiculopathy vs LBP, especially in areas involving intersegmental control of degenerative spondylolisthesis (at levels L4-L5 and L5-S1).

Matsunaga [23] published a follow-up at 10-18 years, and found that the increase in the degree of spondylolisthesis did not correlate with neurologic deterioration. The only symptom that did change with intervertebral disc collapse was an improvement in LBP; however, no ratings on the functional impact were reported, only perception of pain.

In the present study, degree of spondylolisthesis was related to functional disability (regardless of spinal stenosis), even in advanced stages of disc degeneration, where we could assume re-stabilization had occurred.

Other associated factors such as thoseproposed by Panjabi should be evaluated in order to be able to explain pain and dysfunction in DS [12]. Carlson [24] has suggested that weakness alone is not enough to cause LBP although muscle imbalances are an important factor.

It is considered that the normal agonist-antagonist ratio in the trunk varies from 1-1.3. Ratios may be increased by higher moments generated by the extensor muscles, that increase axial load vectors transmitted to zigapophyseal joints. We believe muscle disturbances like this, may underlie the functional disability described in this study.

On the other hand, it has also shown that patients with chronic LBP have abnormal reflexes for intrinsic muscle activation $[25,26]$ which may explain why regardless of the strength capacity of local muscles, segmental stability may be affected if they are not activated at the appropriate time, a factor that we will take into account in our future studies.
With the present study it can be concluded that muscle trunk imbalance with predominance of extensor over flexors muscles is associated with functional disability. We therefore propose that rehabilitation programs should be designed to improve muscle balance rather than muscle strength alone. Further studies should be conducted to confirm these findings.

\section{References}

[1] Rosenberg NJ. Degenerative spondylolisthesis: predisposing factors. J Bone Joint Surg Am. 1975;57:467-74.

[2] Matsunaga S, Sakou T, Morizono Y, et al. Natural history of degenerative spondylolisthesis: pathogenesis and natural course of the slippage. Spine. 1990;15:1204-10.

[3] Sengupta DK. Herkowitz HN. Degenerative spondylolisthesis: Review of current trends and controversies. Spine. 2005; 30:S71-S81.

[4] Love TW, Fagan AB, Fraser RD. Degenerative spondylolisthesis: Developmental or acquired? J Bone Joint Surg Br. 1999;81:670-4.

[5] Takahashi K, Miyazaki T, Takino T, et al. Epidural pressure measurements: relationship between epidural pressure and posture in patients with lumbar spinal stenosis. Spine. 1995;20:650-3.

[6] Takahashi K, Kagechika K, Takino T, et al. Changes in epidural pressure during walking in patients with lumbar spinal stenosis. Spine. 1995;20:2746-9.

[7] Brisby H. Nerve root injuries in patients with chronic low back pain. Orthop Clin North Am. 2003;34:221-30.

[8] Jenis LG, An HS. Spine update: lumbar foraminal stenosis. Spine. 2000;25: 389-94

[9] Mooney V, Robertson J. The facet syndrome. Clin Orthop. 1976;115:149-56.

[10] Dreyfuss PH, Dreyer SJ. Lumbar zygapophysial (facet) joint injections. Spine J. 2003;3(suppl):50-9.

[11] Panjabi M, Abumi K, Duranceau J, et al. Spinal stability and intersegmental muscle forces. A biomechanical model. Spine. 1989;14:194-9.

[12] Panjabi MM. The stabilizing system of the spine. Part 1. Function, dysfunction adaption and enhancement. J Spinal Disord. 1992;5:383-9.

[13] Faries M, Greenwood M. Core training: stabilizing the confusion. National Strength and Conditioning Association. 29(2):10-25.

[14] Suni J, Rinne M, Natri A, et al. Control of the lumbar neutral zone decreases low back pain and improves self-evaluated work ability a 12-month randomized controlled study. Spine. 2006;31(18):E611-E620

[15] O'Sullivan PB, Twomey T, Allison GT. Evaluation of specific stabilizing exercise in the treatment of chronic low back pain with radiologic diagnosis of spondylolysis or spondylolisthesis. Spine. 1997;2(24):2959-2967.

[16] Fairbank JCT, Pynsent PB. The oswestry disability index. Spine. 2000;25(22):2940-2953.

[17] Thompson N, Gould GJ, Davies DE, Ross S. Descriptive measures of isokinetic trunk testing. J Orthop Sports Phys Ther. 1985;7:43-49.

[18] Kjaer P, Bendix T, Sorensen JS, et al. Are MRI-defined fat infiltrations in the multifidus muscles associated with low back pain? BMC Medicine. 2007;5:2. 
[19] Pfirrmann C, Metzdorf A, Zanetti M, et al. Magnetic resonance classification of lumbar intervertebral disc degeneration. Spine. 2001;26:1873-1878.

[20] Kalichman L, Hunter D. Diagnosis and conservative management of degenerative lumbar spondylolisthesis. Eur Spine J. 2008; 17:323-335.

[21] Wallwork TL, Stanton WR, Freke M, Hides JA. The effect of chronic low back pain on size and contraction of the lumbar multifidus muscle. Man Ther. 2009 Oct;14(5):496-500.

[22] Hyun JK, Lee JY, Lee SJ, Jeon JY. Asymmetric atrophy of multifidus muscle in patients with unilateral lumbosacral radiculopathy. Spine. 2007;32(21):E598-602.
[23] Matsunaga S, Ljiri K, Hayashi K. Nonsurgically managed patients with degenerative spondylolisthesis: a 10- to 18year follow-up study. Journal of Neurosurgery: Spine. 2000; 93(2):194-198.

[24] Carlson C. Curr Rev Musculoskelet Med. 2009;2(2):88-93.

[25] Moseley GL, Hodges PW. Are the changes in postural control associated with low back pain caused by pain interference? Clin J Pain. 2005;21(4):323-9.

[26] Moorhouse K, Granata K. Role of reflex dynamics in spinal stability: intrinsic muscle stiffness alone is insufficient for stability. J Biomech. 2007;40(5):1058-1065. 